\title{
Mathematics anxiety - where are we and where shall we go?
}

Krzysztof Cipora ${ }^{1 *}$, Flavia H. Santos ${ }^{2}$, Karin Kucian ${ }^{3}$ \& Ann Dowker ${ }^{4}$

${ }^{1}$ Centre for Mathematical Cognition, Loughborough University, LE11 3TU, United Kingdom; k.cipora@lboro.ac.uk

2 UCD Music and Math Cognition, School of Psychology, University College Dublin, D04 V1W8, Ireland; flavia.santos@ucd.ie

${ }^{3}$ Center for MR-Research, University Children's Hospital Zurich, Switzerland; karin.kucian@kispi.uzh.ch

${ }^{4}$ Department of Experimental Psychology, Oxford University, New Radcliffe House, Radcliffe Observatory Quarter, Woodstock Road, Oxford OX2 6GG, United Kingdom; ann.dowker@psy.ox.ac.uk

* Corresponding author

Published in Annals of the New York Academy of Sciences. Please refer to the final Open Access version available on the journal website:

https://nyaspubs.onlinelibrary.wiley.com/doi/full/10.1111/nyas.14 $\underline{770}$ 


\begin{abstract}
In this paper we list ten fairly undisputed claims about Mathematics Anxiety (MA) and propose where MA research should focus including theoretical clarifications on what MA is, and what constitutes its opposite pole, discussion on construct validity, specifically relations between self-descriptive, (neuro)physiological and cognitive measures; discussion of the discrepancy between state and trait MA and theoretical and practical consequences; discussion of prevalence of MA and need for establishing external criteria for estimating prevalence and proposal of such criteria, and exploration of the effects of MA in different groups, such us highly anxious and high math-performing individuals; classroom applications of MA knowledge; the effects of MA outside educational settings; and the consequences of MA on mental health and well-being.
\end{abstract}




\section{Where are we?}

Mathematics anxiety (MA) has been investigated in the past seven decades ${ }^{1}$ in its psychometric, experimental, behavioral and neurophysiological aspects. As one might expect, studies conducted for such a long period of time brought several insights into the nature of this phenomenon. Luckily, most of these developments have been thoroughly summarized in several review papers and meta-analyse ${ }^{2-8}$. Thus, we do not aim here to duplicate this vast amount of work on research synthesis but rather to direct an interested reader to specific papers. We also do not wish to add another experimental study to this volume. We limit ourselves to summarizing relatively undisputed claims about MA (cf. Box 1) and try to point to open questions and areas, which, in our opinion, deserve more attention in future research.

\section{BOX 1. What we know about Mathematics anxiety}

1. MA exists - it cannot be reduced to other constructs ${ }^{3,9,10}$ :

a. MA is distinct from other types of anxiety (state anxiety, trait anxiety, test anxiety), but correlates with them positively with moderate effect sizes ${ }^{9,10}$.

b. MA is distinct from low mathematics performance ${ }^{5,8,9,11,12}$, but MA and performance are consistently found to be negatively correlated, at least in secondary pupils and adults, though usually with low to moderate effect sizes $3,5,7-9,12$.

2. MA is independent of mathematical learning disorder / developmental dyscalculia, however, there are some comorbidities between the two ${ }^{13}$.

3. MA is present across several stages of development starting from early elementary school and increases in frequency until adulthood ${ }^{5,7,12}$.

4. MA can be reliably measured with self-descriptive instruments ${ }^{14}$.

5. MA is not a unidimensional construct, although there is no agreement concerning the dimensions. Different models assume different factor structure. Most common components refer to anxiety related to being tested in mathematics and anxiety related to learning mathematics. Another factor commonly mentioned refers to anxiety experienced in daily life situations ${ }^{14}$. Other authors differentiate between cognitive, emotional, and physiological components ${ }^{14}$. 
6. MA is linked to long-term career choices such that individuals with elevated MA are less likely to pursue math-intense STEM careers ${ }^{9,10}$.

7. MA can be observed in various cultural and linguistic contexts ${ }^{3,15,16}$. However, most studies were conducted in developed countries.

8. Girls / women typically score higher in MA than boys / men, but this difference is nowadays usually not reflected in mathematics performance ${ }^{5,9,17}$. This effect is present in several cultures ${ }^{18}$, however, the exact reasons for that difference remain unclear.

9. Several neural (e.g., increased activation of brain regions associated with emotional response, such as amygdala or insula), physiological (e.g., skin conductance responses when solving arithmetic problems), and cognitive correlates (e.g., reaction times and accuracies in elementary number processing tasks) of MA have been identified ${ }^{4,19,20}$.

10. It is possible to alleviate MA, which can also lead to an increase in mathematics performance. Behavioral and cognitive-behavioral therapies have most commonly been successful ${ }^{6,9}$.

\section{Blank spots and the future research agenda}

\section{How do we understand MA?}

An attentive reader might have noticed that in the previous part, unlike most papers, we have not defined MA. We avoided doing so for a purpose. We think that the differences between definitions provided by different authors are not just about wording. On the contrary, they might reflect different understanding of MA and may at least to some extent contribute to misunderstandings between researchers, and between researchers and practitioners.

One group of definitions seems to treat MA as a trait or state that can vary between individuals and between situations. For instance, Richardson and Suinn ${ }^{21}$ define MA as $A$ feeling of tension and anxiety that interferes with the manipulation of numbers and the solving of mathematical problems in (...) ordinary life and academic situations. Similarly, Ashcraft and Ridley ${ }^{10}$ define it as Negative states related to mathematics and mathematical situations. The other group of definitions can be considered more "clinical." For instance, according to Lazarus $^{22}, \mathrm{MA}$ is An irrational and impeditive dread of mathematics. Tobias ${ }^{23}$ defines MA as The panic, helplessness, paralysis and mental disorganization that arises among some people when they are required to solve a mathematical problem. According to classical studies by Faust, MA meets the criteria for specific phobia ${ }^{10}$. This claim is also supported by more recent 
study showing that behavioral and brain activation patterns of highly math anxious individuals when being exposed to mathematical problems (not even being required to solve them) resembles patterns observed in individuals with other phobias being presented with phobiarelated stimuli ${ }^{24}$. However, currently MA is not described in medical or psychiatric manuals and is not viewed as requiring pharmacological treatment.

Recently Ashcraft postulated that MA can be seen as personality construct, cognitive construct, sociocultural construct, or neuro-biological construct, which means that MA might be defined from even more perspectives ${ }^{25}$. In our view, all types of definitions are useful and valid. One could say that they are, in fact, very similar because the "clinical" ones are just characterizing individuals with extremely high MA. On the other hand, such definitions might imply that only extremely high MA is detrimental to mathematics performance and / or individuals' well-being. They might also imply the presence of qualitative differences between anxious and non-anxious individuals, which, as we show in the following sections, seems not to be the case. We are yet to understand the level, at which MA becomes detrimental to people's performance and/or well-being. At the same time, we propose that when conducting future research or synthesizing existing studies, researchers consider different understanding of $\mathrm{MA}$, and mind (potentially implicit) assumptions about its nature originating from different research traditions.

Secondly, as far as there is a consensus on what characterizes high MA, there is relatively little research on the opposite pole of the MA spectrum. We can think of different possible scenarios of how the opposite pole of the MA might be: (1) being neutral about mathematics; (2) lack of anxiety but having other negative emotions towards mathematics - anger, hate, dislike ${ }^{26}$; (3) lack of anxiety but resignation and helplessness; (4) feeling positive emotions towards mathematics.

The answer to this question is non-trivial. Looking at other similar constructs, we can, on the one hand, see that, e.g., in the case of general anxiety, the opposite pole is characterized by lack of anxiety. However, in other domains, we can imagine two qualitatively different poles. We certainly know that some individuals reveal positive feelings towards mathematics (e.g., because usually the ones who decide to pursue their careers in mathematics are passionate about it) and a few studies have looked directly at the influence of positive emotions on mathematical performance $27-29$. However, it remains uncertain whether these positive feelings constitute the opposite pole of anxiety, or whether the two can be independent. We 
also do know that some individuals experience other negative emotions when encountering mathematics, and have negative attitudes towards this subject, even if those emotions and attitudes are not directly related to anxiety: e.g., dislike, boredom, and perception of the subject as irrelevant or useless. Nevertheless, such feelings might still co-occur with or even contribute to MA in some individuals, so this aspect needs a more thorough investigation. To sum up, being clear about definitions and exploring the whole spectrum of MA might help integrate existing theories of MA. According to a very well-known cliché, there is nothing more practical than a good theory. We believe that in the case of MA this cliché is valid: better understanding what is MA, whom it affects and where it ends can guide interventions both in respect of whom we target [e.g., only extremely highly anxious, "(sub-)clinical"-like groups, or also moderately anxious, "non-clinical" individuals]. It can also help to define g the desired goal of the intervention - making participants neutral towards mathematics, making them actively like it, or maybe not bothering (at least within the MA intervention) about other negative feelings such as boredom and only alleviating the anxiety. It may also be that interventions should not target MA as such, but should focus on other aspects, e.g., resilience or mathematical self-efficacy, which while related to MA, remain distinct from it (see section below).

\section{Construct validity}

So far, most studies showing construct validity of MA focus on data coming from selfdescriptions. They show that the theoretically proposed structure (e.g., components related to learning mathematics, being tested in mathematics, or encountering mathematics in everyday life, see ${ }^{14}$ for a review) is reflected in the factor structure observed in the data. At the same time, a recent study by Pizzie and Kraemer ${ }^{30}$ raises an important issue of overlap between MA and Test anxiety. This is especially visible in case of the MARS questionnaire ${ }^{31}$. The instrument they developed was aimed at differentiating anxiety types. While this goal was achieved, it needs to be investigated whether the differentiation between MA and Mathematics Self-Concept, Mathematics Self-Efficacy ${ }^{16}$, and Mathematics attitudes remains. Importantly, the differences between MA measurement instruments should be accounted for when investigating links between MA and mathematics performance ${ }^{30}$. What also needs to be addressed is the relation between MA and statistics anxiety, which has gained interest in recent years ${ }^{32-34}$. In particular, the similarity of items measuring these two constructs calls for 
more studies to clarify relations between them. There are known to be some differences in the findings for mathematics anxiety and statistics anxiety: in particular, gender differences in anxiety are not as consistently found for the latter ${ }^{35,36}$. Additionally, the links between statistics anxiety and performance in statistics (if present at all) are weaker than those between MA and mathematics performance ${ }^{33}$ but see $\mathrm{s}^{5,37}$.

Going beyond self-descriptions, studies using other measures [(neuro)physiological, cognitive] in the context of MA are relatively scarce and rather focus on group level differences. Studies providing converging evidence from psychometric, (neuro)physiological, and cognitive data remain scarce ${ }^{38-44}$. Despite their relative difficulty in comparison to questionnaire administration, such studies might bring new insights into our understanding of MA. They might prove particularly useful, as compared to self-descriptions as they not only refer to some previous or current experience but allow tracing the (neuro)physiological / cognitive response while performing anxiety-inducing mathematics tasks. However, evidence so far shows that questionnaires remain more reliable measures of MA than implicit MA measures ${ }^{45}$. Some questions that need to be answered are whether the correlation between self-descriptive and (neuro)physiological / cognitive measures hold for all individuals, and whether these correlations are present when we consider other anxiety types. Looking into this might shed new light on why in some participants, MA (defined as scores in MA questionnaires) is linked to lower performance and avoidance of mathematics while in some it is not.

\section{State trait discrepancy}

The state-trait distinction is crucial when thinking of general anxiety ${ }^{46}$. Recent years have brought interesting evidence that considering this distinction for MA ${ }^{47-50}$ might deepen our theoretical understanding. Additionally, such a strong theory can again turn out very practical when it comes to MA measurement and guiding interventions. Potentially, it might be easier to target the state anxiety than the trait characteristic. Teaching people (both children / students in an educational context, but also adults who are no longer receiving formal education) strategies on how not to allow state anxiety to arise might turn to be a useful way for alleviating the negative effects of MA. We believe that work on state and trait aspects of MA could be very fruitful in terms of both theory building and practical implications. 


\section{Who is math anxious, or MA prevalence}

When discussing MA with non-experts, policymakers, or educational practitioners, we often receive a completely reasonable question about the prevalence of MA. Despite being very fundamental, this question still does not have a straightforward and satisfactory answer. As in psychological diagnostics in general, we can think of several criteria for estimating MA prevalence. Looking at distributions of different MA scale scores, we see that they either follow the normal distribution (i.e., most participants reveal scores close to sample mean and scores further away from the mean are less and less frequent), or their distribution is rightskewed (most frequent are the relatively low scores, and higher scores are less and less frequent cf. Figure 1). In any case, there are no indications that the distribution is bimodal (i.e., two relatively separate groups of scores, low, high ones; see ${ }^{51,52}$ ).

Thus, there is no qualitative difference between anxious and non-anxious individuals and no natural differentiation between them. Quite often in psychology, a statistical criterion is used classifying individuals who score within a certain percentile on the scale as anxious. While useful for the purpose of the individual diagnosis (e.g., we can say that an individual has MA higher than $95 \%$ of the population), this does not solve all the problems. First, the cutoff is arbitrary, and there is no consensus among researchers as to which value should be used (someone being in the $94^{\text {th }}$ percentile might still benefit from the intervention and does not differ much from someone in the $96^{\text {th }}$ percentile). Secondly, the cutoff values depend strongly on the quality and representativeness of the normative sample. The problem with the statistical criterion gets even more pronounced when we want to say something about the prevalence of the MA. When a statistical criterion is used (e.g., the top 10\%), saying that the prevalence of MA is $10 \%$ reveals circular reasoning as it simply reiterates the adopted arbitrary statistical cut-off criterion. The other problem of such an approach is the external validity of such criteria. In other words, our conclusion on whether someone is math anxious or not (even aside from attempts to estimate the prevalence of $\mathrm{MA}$ ) is solely based on the distribution of the scores in the population (approximated by means of the sample), but it does not tell much about whether and how this MA level affects mathematical performance or well-being. Thus, future studies should focus on developing better diagnostic / cutoff criteria. This should not be limited to creating widely accepted and solid norms but should also consider external criteria for MA diagnosis. 
A Simulated (bimodal) distribution

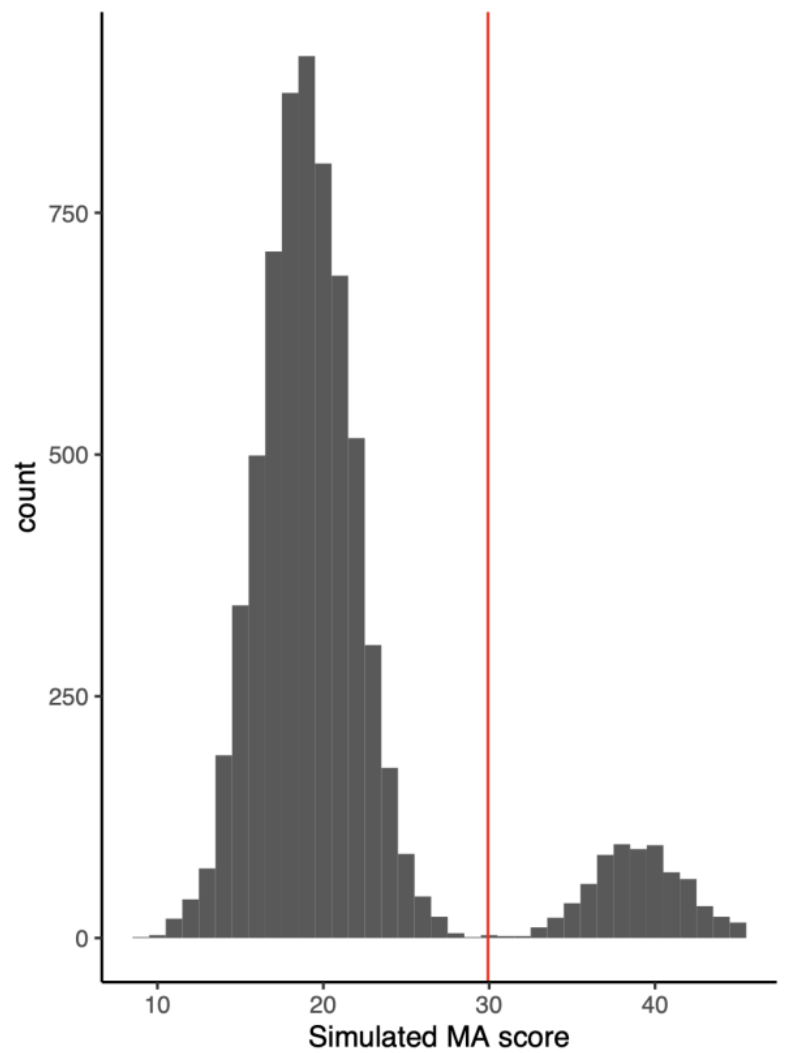

B Empirical normal/right-skewed distribution

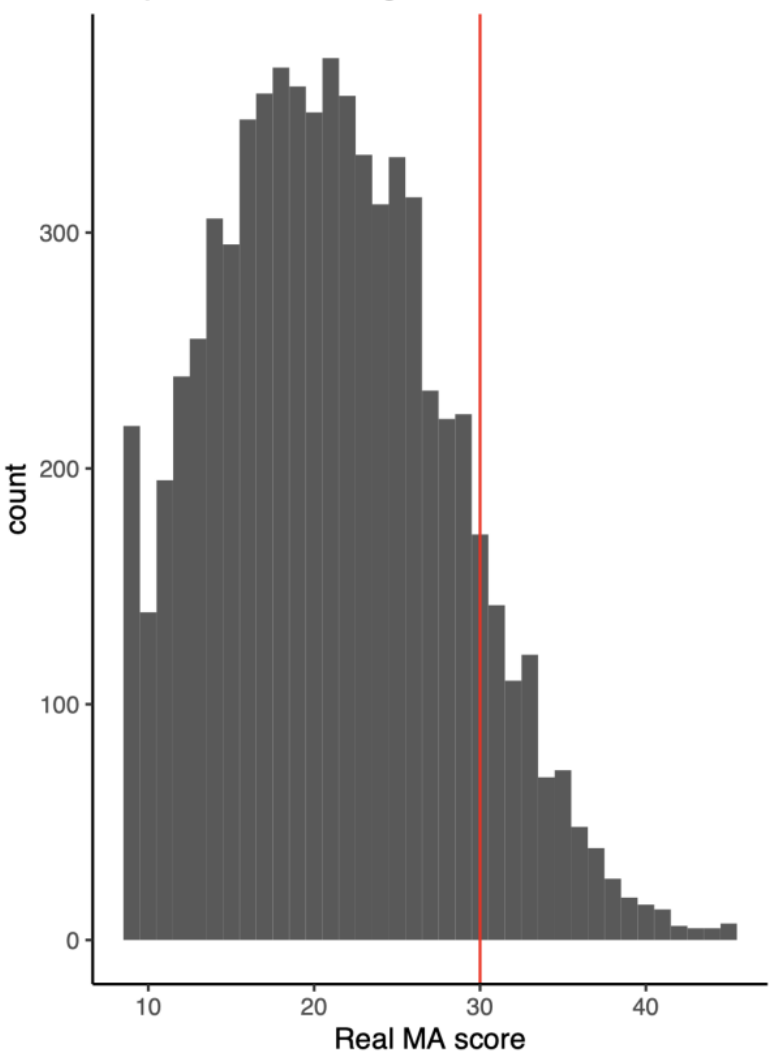

Figure 1. Panel A: Simulated bimodal distribution (7006 observations, possible range 9-45; matching the data presented in Panel B). If MA scores distribution revealed two distinct groups, establishing cut-off criteria would be straightforward. Panel B: Empirically observed distribution of raw scores of MA questionnaire AMAS $^{53} ; n=7006$, possible values ranging from 9 to 45 with higher values corresponding to higher anxiety) is closer to normal / rightskewed distribution. In this case, we cannot find any natural cut-off. Empirical data taken from the database by Cipora \& Caviola (DOI 10.17605/OSF.IO/QYS6N). In both panels red vertical lines represent hypothetical cut-off of top $10 \%$. The figure, the AMAS data, and the R code used to generate it are available at: https://osf.io/ribwm/. Figure by K. Cipora et al., 2022; available at https://doi.org/10.17605/OSF.IO/RJBWM under a CC-BY4.0 license.

Use of external criteria can already be found in early MA studies. For instance, Richardson and Suinn ${ }^{21}$ proposed that about $11 \%$ of the university student population's MA is high enough that they are likely to need some counselling. In general, prevalence estimates available in the literature differ greatly starting from $2 \%$ and going up to as much as $68 \%$ (see 3 for an overview). Some authors, e.g., ${ }^{54}$ look at how many individuals report experiencing negative emotions while doing mathematics. Such an "external" criterion of feeling discomfort while doing mathematics can be useful in some context as a cutoff. However, one 
might argue that such subjective measures should be avoided in favor of more objective ones. We propose that following criteria should be checked for MA diagnosis and intervention planning: (1) whether the MA is affecting mathematics performance beyond the current skill level [for instance the discrepancy between math performance in relaxed (e.g., self-paced) and a stressful (e.g., under time pressure, high stakes) situation] ${ }^{42,55,56}$, but this method may not be useful in case of individuals characterized by very high MA levels, (2) to which extent avoidance of mathematics was a decisive factor for life / vocation choices (in case of adolescent / adult assessment), (3) to which extent dealing with mathematics is associated with subjective discomfort and unpleasant feelings (4) whether the problem remits spontaneously or pertains for a specific period of time (similar to diagnosis of other forms of anxiety). We postulate that fulfilling any of the criteria 1-3 in conjunction with their persistence in time should be sufficient for a diagnosis. Please mind that at this stage this is only a tentative proposal, and specific thresholds for each of them are yet to be established. This tentative list of criteria should be further supplemented by results of qualitative studies aimed at understanding potential impacts of MA from personal perspectives. Such a qualitative evaluation might become a part of the individual MA diagnosis. Having representative data regarding proportions of population that meet such criteria will provide new insights into the prevalence of MA and the scale of the impact it has on society above and beyond chasing our own tail by looking solely at statistical criteria.

\section{Exploring the neglected parts of MA - performance scatterplot}

As we already discussed, there is a clear link between MA and mathematics performance with an average effect size of about -.3. Needless to say, that this correlation is at best "moderate," and this value indicates that the common variance of MA and mathematics performance is below $10 \%$. Nevertheless, it seems that most studies were focused on extremes of this scatterplot - individuals scoring high in MA and low in mathematics and those scoring low in MA and high in mathematics. However, what remains largely unknown are other parts of this scatterplot.

Firstly, we do not know much about individuals who score around average with respect to both variables. To what extent is their mathematics performance affected by MA? Some evidence shows that MA predicts lower grades in STEM courses, even after controlling for mathematical abilities ${ }^{57}$ leading us to consider the extent to which MA affects life choices and 
well-being. Answering these questions would help us understand whether and to what extent addressing MA within untargeted programs aimed at general (unselected) populations is justifiable, and whether / what kind of benefits such programs could bring.

Secondly, there are individuals who perform well or even very well despite their (very) high math anxiety. For instance, Devine et al. ${ }^{13}$ found that around $80 \%$ of secondary schoolers scoring above the $90^{\text {th }}$ percentile in $\mathrm{MA}$, were scoring within the typical range in mathematics (-1SD and higher). Several studies have shown that the link between MA and mathematics performance is not uniform across different groups (a review $\mathrm{in}^{14}$ ), with some studies suggesting that motivation is linked more strongly to mathematics performance than MA is ${ }^{58,59}$ and other studies showing more complex patterns ${ }^{60}$. Self-concept with regard to mathematics also interacts to a significant degree with the relationship between MA and mathematics performance ${ }^{28}$. In addition, some STEM / mathematics students and professionals score above the minimum level in MA (e.g. $\left.{ }^{61}\right)$. Despite MA, these individuals are successfully pursuing STEM careers. It is worth understanding the factors that may contribute to this. Additionally, it would be important to know whether (a) mathematics performance of these individuals is still affected (i.e., if not MA they would be scoring even higher)? (b) MA is affecting their well-being (and whether they might benefit from support in this area)? (c) there are specific factors, which help them pursue a career in STEM despite elevated MA (and whether knowing these factors could be used to support other highly math anxious individuals)?

Conversely, we do not know much about individuals who are scoring low in MA despite very low mathematics performance (e.g., some children with mathematical learning disorder / developmental dyscalculia ${ }^{13}$ ). We do not know what prevented them from developing MA within the vicious circle ${ }^{2}$ of MA being amplified by repeated failures in mathematics. Understanding these mechanisms can also inform the development of methods for supporting individuals with specific learning disorders, so that apart from receiving specific support in the domain of mathematics, they might also be protected from developing MA. Studying such individuals might also increase our understanding of the role of motivation: it might turn out that in some cases both low mathematical attainment and low MA might be caused by regarding mathematics as unimportant or uninteresting, a phenomenon which needs to be distinguished from low mathematical attainment associated with dyscalculia or similar problems. 
In general, we postulate that to understand MA, we should build a more thorough picture by focusing on specific groups of individuals, not only those "driving" the correlation between MA and mathematics performance, who were in the focus of the attention in past decades.

\section{How to apply it to classroom practice and educational policy?}

From our own experience, we know that mathematics teachers are well aware that some of their students are anxious about mathematics. At the same time, we see that teachers really want to address this problem and learn from the researchers. Thus, we believe that one of the biggest challenges of MA research is bringing the knowledge accumulated in past decades to the classroom practice in a scalable way. This should not be limited to "raising awareness" and similar initiatives, which are of course helpful, but do not offer ready solutions. At the same time, it is important to avoid simplistic explanations and solutions, which sometimes appear in the public discourse (e.g., that MA is predominantly caused by some single factor). Developing proper resources, which could be used by teachers is one of the biggest challenges to the field. On top of raising awareness of the teachers and education practitioners, we need proper screening tools to be administered to children, which are easy to use, valid, and reliable. That would allow teachers to identify pupils who are in the process of developing MA, before their anxiety starts to affect their well-being significantly and/or a vicious cycle develops between lowered mathematical performance and increased MA. This part, despite being challenging, is relatively simple to implement. The real difficulty is in finding ways to develop scalable and cost-effective intervention programs, especially those targeted at emotional regulation and grounding, before approaching mathematics problems. We know that psychotherapy-inspired interventions can prove effective for alleviating $M A^{9}$. Unfortunately, such interventions typically require one-to-one contact with a highly trained professional, thus they incur considerable costs and are not easily implemented. Several brief interventions at the school aimed at alleviating MA have been tested (e.g., ${ }^{62,63}$ ). However, the effects of such interventions have not always been replicated ${ }^{64}$. It seems reasonable to think of something in between individual psychotherapy-based interventions and brief one-time interventions (see ${ }^{62,63}$ ). Such programs should ideally be administered by the teacher / educational practitioner: preferably within integrated mathematics curricula, which focus also on prevention and remediation of MA. Developing such programs should be guided by the conclusions of studies on who are likely to benefit from MA interventions (see section 
above). Some important considerations for development of such interventions can be found $\mathrm{in}^{65}$. It seems that game-based interventions might be an interesting avenue of investigation ${ }^{66}$.

The development of such programs would certainly benefit from a constant exchange of information between researchers and practitioners. Such collaborations should be fostered both at the local and global levels so that researchers have access to "reality checks" on whether solutions they propose are feasible and applicable in a wider range of contexts.

The initiatives should not be limited to children. Early years education teachers often reveal relatively high $M A^{67-70}$ and this may increase $M A$ in their pupils ${ }^{71}$. Therefore, $M A$ intervention programs should also be developed for this group. Intervention programs should also be available to parents, as their attitudes and behaviors towards mathematics and their MA may also negatively affect their children' learning experience ${ }^{72,73}$.

\section{Outside educational settings}

Most MA studies have been conducted in educational settings (schools, colleges, universities). Even those that do not test current pupils/ students have usually involved individuals, who were linked to some educational settings: elementary school teachers ${ }^{67,71}$, or parents of elementary school children (e.g., ${ }^{72-74}$ ). Studies investigating adults outside the educational system are relatively scarce (with the notable exception of Hart and Ganley ${ }^{52}$ ).

On the other hand, several models of MA explicitly consider that MA can manifest itself in daily life situations (calculating the change, tip, etc. ${ }^{75-77}$ ). At the same time, we are aware of serious economic costs of low numeracy both at the individual and global level ${ }^{78,79}$. For this reason, it would be worthwhile to focus on individuals who have already left education to see their MA level, its consequences, and potential means for remediation. The latter have not, to the best of our knowledge, been investigated in these groups. One of the very few studies investigating such a population ${ }^{52}$ has in general replicated findings from studies conducted within educational settings. Nevertheless, we believe that still a lot needs to be done to better understand MA outside academic context and possibilities for addressing it.

\section{Links to well-being}

Most studies looking at the consequences of MA focused on its links to mathematics performance, vocation choice, etc. Relatively less attention was given to consequences on individual well-being and mental health. We know that anxiety correlates negatively with 
well-being ${ }^{80}$. It would be worth investigating similar long-term costs of MA including mental health and quality of life ${ }^{81-83}$. We also do not know whether in individuals who decide on pursuing mathematics-related careers despite elevated MA, such a career happens at the expense of quality of life and well-being. Chronic stress can be a serious risk factor for burnout and potentially other mental and physical health conditions ${ }^{84}$, and withdrawal from specific careers (which seems to be more prevalent among women) ${ }^{85}$. Moreover, even for people who do not pursue mathematics-related careers, there is an increasing need in the modern world to take responsibility for one's own budgeting, for example in online banking and often in the need to make decisions about the details of pensions. Such demands may increase the stress of MA even in those who can cope cognitively with them, as well as leading to financial problems and social exclusion and their effects on well-being for those who cannot. Another group which may experience MA impact on well-being are individuals, who decided not to pursue their passion within STEM (e.g., in engineering or architecture), because it comprises math. We strongly believe that focusing research on the links of MA to well-being will bring several theoretical and practical benefits.

\section{BOX 2. Pending questions in Mathematics Anxiety research}

1. How should MA be understood? Do we look at it from a clinical-like perspective, or we treat it as a differential variable?

2. What is the empirical support for the validity of the MA as a construct: (a) convergence between self-descriptive, implicit, and physiological MA measures, (b) relations between mathematics testing (evaluation) anxiety and test anxiety, (c) relations between MA and statistics anxiety?

3. Can focusing on the state-trait discrepancy in the MA foster theoretical understanding of MA and intervention planning?

4. What is the prevalence of MA, and which external criteria should be used to avoid estimating the prevalence based purely on statistical criteria?

5. What can be learned by looking at individuals, who perform high in mathematics and score high in MA, and from those whose MA is low despite low mathematics performance? Could such discrepancies be influenced by cultural factors as well as by individual differences and local environmental factors? 
6. How might MA and its relationship to mathematical performance interact with intrinsic and extrinsic motivation for mathematics?

7. How can MA research be applied in classroom practice and educational policy; in particular, how can scalable interventions targeting MA be prepared?

8. What happens to MA outside educational settings, what are its consequences; and how can they be alleviated?

9. What are the consequences of MA beyond those on mathematics performance, especially whether and how does it impact individuals' well-being?

10. When and how does MA begin? To what extent can it be seen in the early school years and perhaps even the preschool years? Could very early interventions prevent it from developing?

\section{Conclusions}

Past years have brought an impressive number of studies on MA. Recent review papers, metaanalyses, and books give a hint on the amount of our current knowledge. We think this was a good moment to reflect not only on what we know but also on what we want to know. We tried to point to areas which deserve research effort. We also summarize them in Box 2 . They span from cleaning and clarifying definitions and theories to bringing these theories to reality checks and practice. When pointing out these blank spots, we by no means wish to undermine the great amount and quality of MA research that has been done so far. Rather on the contrary, we are truly optimistic that these blank spots will soon be filled with solid theoretical and empirical work, and eagerly wait to witness these developments.

\section{Author contributions}

KC wrote an initial draft. It was critically reviewed, complemented and discussed by FHS, KK, and AD. All authors approved the final version of the manuscript.

\section{Acknowledgements}

KC is funded by Research England via the Centre for Mathematical Cognition. We are grateful to the Editors of Annals of the New York Academy of Sciences for agreeing to host this Special Issue. We would also like to thank Mateusz Hohol for reading an early draft of this paper. 


\section{References}

1. Dreger R.M. \& Jr. Aiken, Lewis R. 1957. The identification of number anxiety in a college population. Journal of Educational Psychology 48: 344-351.

2. Carey E., F. Hill, A. Devine, et al. 2016. The chicken or the egg? The direction of the relationship between mathematics anxiety and mathematics performance. Frontiers in Psychology 6: 1-6.

3. Dowker A., A. Sarkar \& C.Y. Looi. 2016. Mathematics anxiety: What have we learned in 60 years? Frontiers in Psychology 7:.

4. Suárez-Pellicioni M., M.I. Núñez-Peña \& À. Colomé. 2016. Math anxiety: A review of its cognitive consequences, psychophysiological correlates, and brain bases. Cognitive, Affective and Behavioral Neuroscience 16: 3-22.

5. Barroso C., C.M. Ganley, A.L. McGraw, et al. 2021. A Meta-Analysis of the Relation Between Math Anxiety and Math Achievement. Psychological Bulletin 147: 134-168.

6. Ramirez G., S.T. Shaw \& E.A. Maloney. 2018. Math Anxiety: Past Research, Promising Interventions, and a New Interpretation Framework. Educational Psychologist 53: 145-164.

7. Zhang J., N. Zhao \& Q.P. Kong. 2019. The relationship between math anxiety and math performance: a meta-analytic investigation. Frontiers in Psychology 10: 1613.

8. Namkung J.M., P. Peng \& X. Lin. 2019. The Relation Between Mathematics Anxiety and Mathematics Performance Among School-Aged Students: A Meta-Analysis: Review of Educational Research 89: 459-496.

9. Hembree R. 1990. The Nature, effects, and relief of Mathematics Anxiety. Journal for Research in Mathematics Education 21: 33-46.

10. Ashcraft M.H. \& K.S. Ridley. 2005. Math anxiety and its cognitive consequences: A tutorial review. In Handbook of Mathematical Cognition Campbell J.I.D., Ed. 315-327. New York: Psychology Press.

11. Ma X. \& N. Kishor. 1997. Assessing the relationship between attitude toward mathematics and achievement in mathematics: A meta-analysis. Journal for Research in Mathematics Education 28: 26-47.

12. Caviola S., E. Toffalini, D. Giofrè, et al. 2021. Math Performance and Academic Anxiety Forms, from Sociodemographic to Cognitive Aspects: a Meta-analysis on 906,311 Participants. Educational Psychology Review 1-37.

13. Devine A., F. Hill, E. Carey, et al. 2018. Cognitive and emotional math problems largely dissociate: Prevalence of developmental dyscalculia and mathematics anxiety. Journal of Educational Psychology 110: 431-444.

14. Cipora K., C. Artemenko \& H.-C. Nuerk. 2019. Different ways to measure math anxiety. In Mathematics Anxiety: What is known and what is still to be understood Mammarella I., Caviola S., \& Dowker A., Eds. 20-41. Routledge.

15. Foley A.E., J.B. Herts, F. Borgonovi, et al. 2017. The Math Anxiety-Performance Link: A Global Phenomenon. Current Directions in Psychological Science 26: 52-58.

16. Lee J. 2009. Universals and specifics of math self-concept, math self-efficacy, and math anxiety across 41 PISA 2003 participating countries. Learning and Individual Differences 19: 355-365.

17. Spelke E.S. 2005. Sex differences in intrinsic aptitude for mathematics and science? A critical review. American Psychologist 60: 950-958. 
18. OECD. 2013. "PISA 2012 Results: Ready to Learn: Students' Engagement, Drive and Self-Beliefs (Volume III)."

19. Artemenko C., G. Daroczy \& H.-C. Nuerk. 2015. Neural correlates of math anxiety - an overview and implications. Frontiers in Psychology 6:.

20. Avancini C. \& D. Szúcs. 2019. Psychophysiological correlates of Mathematics Anxiety. In Mathematics Anxiety: What is known and what is still to be understood Mammarella I., Caviola S., \& Dowker A., Eds. 42-61. Routledge.

21. Richardson F.C. \& R.M. Suinn. 1972. The Mathematics Anxiety Rating Scale: Psychometric data. Journal of Counseling Psychology 19: 551-554.

22. Lazarus M. 1974. Mathophobia: Some Personal Speculations. National Elementary Principal 53: 16-22.

23. Tobias S. 1978. "Overcoming Math Anxiety." Houghton Mifflin Company.

24. Pizzie R.G. \& D.J.M. Kraemer. 2017. Avoiding math on a rapid timescale: Emotional responsivity and anxious attention in math anxiety. Brain and Cognition 118: 100107.

25. Ashcraft M.H. 2019. Models of Math Anxiety. In Mathematics Anxiety: What is known and what is still to be understood Mammarella I., Caviola S., \& Dowker A., Eds. 1-19. Routledge.

26. Larkin K. \& R. Jorgensen. 2016. 'I Hate Maths: Why Do We Need to Do Maths?' Using iPad Video Diaries to Investigate Attitudes and Emotions Towards Mathematics in Year 3 and Year 6 Students. International Journal of Science and Mathematics Education 14: 925-944.

27. Villavicencio F.T. \& A.B.I. Bernardo. 2016. Beyond Math Anxiety: Positive Emotions Predict Mathematics Achievement, Self-Regulation, and Self-Efficacy. Asia-Pacific Education Researcher 25: 415-422.

28. van der Beek J.P.J., S.H.G. van der Ven, E.H. Kroesbergen, et al. 2017. Self-concept mediates the relation between achievement and emotions in mathematics. British Journal of Educational Psychology 87: 478-495.

29. Pinxten M., H.W. Marsh, B. de Fraine, et al. 2014. Enjoying mathematics or feeling competent in mathematics? Reciprocal effects on mathematics achievement and perceived math effort expenditure. British Journal of Educational Psychology 84: 152174.

30. Pizzie R.G. \& D.J.M. Kraemer. 2019. The Academic Anxiety Inventory: Evidence for dissociable patterns of anxiety related to math and other sources of academic stress. Frontiers in Psychology 9:.

31. Richardson F.C. \& R.M. Suinn. 1972. The Mathematics Anxiety Rating Scale: Psychometric data. Journal of Counseling Psychology 19: 551-554.

32. Baloğlu M. 2004. Statistics anxiety and mathematics anxiety: some interesting differences. Educational Research Quarterly 27: 38-48.

33. Macher D., I. Papousek, K. Ruggeri, et al. 2015. Statistics anxiety and performance: Blessings in disguise. Frontiers in Psychology 6: 1116.

34. Trassi A.P., S.J. Leonard, L.D. Rodrigues, et al. 2022. Mediating Factors of Statistics Anxiety in University Students: A Systematic Review and Meta-Analysis. Annals of the New York Academy of Sciences.

35. Mji A. 2009. Differences in university students' attitudes and anxiety about statistics. Psychological Reports 104: 737-744. 
36. Bui N.H. \& M.A. Alfaro. 2011. Statistics anxiety and science attitudes: age, gender, and ethnicity factors. College Student Journal 45: 573-586.

37. Primi C. \& F. Chiesi. 2018. The role of Mathematics Anxiety and Statistics Anxiety in learning statistics. In Looking back, looking forward. Proceedings of the Tenth International Conference on Teaching Statistics Sorto M.A., White A., \& Guyot L., Eds. 1-6. International Statistical Institute.

38. Hartwright C.E., C.Y. Looi, F. Sella, et al. 2018. The Neurocognitive Architecture of Individual Differences in Math Anxiety in Typical Children. Scientific Reports 8:

39. Kucian K., U. McCaskey, R. O'Gorman Tuura, et al. 2018. Neurostructural correlate of math anxiety in the brain of children. Translational Psychiatry 2018 8:1 8: 1-11.

40. Maloney E.A., E.F. Risko, D. Ansari, et al. 2010. Mathematics anxiety affects counting but not subitizing during visual enumeration. Cognition 114: 293-297.

41. Maloney E.A., D. Ansari \& J.A. Fugelsang. 2011. The effect of mathematics anxiety on the processing of numerical magnitude. Quarterly Journal of Experimental Psychology 64: 10-16.

42. Ashcraft M.H. \& E.P. Kirk. 2001. The relationships among working memory, math anxiety, and performance. Journal of Experimental Psychology: General 130: 224-237.

43. van Dijck J.-P., W. Fias \& K. Cipora. 2022. Spatialization in working memory and its relation to math anxiety. Annals of the New York Academy of Sciences.

44. Starling-Alves I., M.R. Wronski \& E.M. Hubbard. 2021. Math anxiety differentially impairs symbolic, but not nonsymbolic, fraction skills across development. Annals of the New York Academy of Sciences.

45. Kucian K., I. Zuber, J. Kohn, et al. 2018. Relation between mathematical performance, math anxiety, and affective priming in children with and without developmental dyscalculia. Frontiers in Psychology 9: 263.

46. Endler N.S. \& N.L. Kocovski. 2001. State and trait anxiety revisited. Journal of anxiety disorders 15: 231-245.

47. Orbach L., M. Herzog \& A. Fritz. 2020. State- and trait-math anxiety and their relation to math performance in children: The role of core executive functions. Cognition 200: 104271.

48. Orbach L., M. Herzog \& A. Fritz. 2019. Relation of State- and Trait-Math Anxiety to Intelligence, Math Achievement and Learning Motivation. Journal of Numerical Cognition 5: 371-399.

49. Roos A.-L., M. Bieg, T. Goetz, et al. 2015. Experiencing more mathematics anxiety than expected? Contrasting trait and state anxiety in high achieving students. High Ability Studies 26: 245-258.

50. Bieg M., T. Goetz, I. Wolter, et al. 2015. Gender stereotype endorsement differentially predicts girls' and boys' trait-state discrepancy in math anxiety. Frontiers in Psychology 6: 1-8.

51. Cipora K., K. Willmes, A. Szwarc, et al. 2017. Norms and validation of the online and paper-and-pencil versions of the Abbreviated Math Anxiety Scale (AMAS) for Polish adolescents and adults. Journal of Numerical Cognition 3: 667-693.

52. Hart S.A. \& C.M. Ganley. 2019. The nature of math anxiety in adults: Prevalence and correlates. Journal of Numerical Cognition 5: 122-139.

53. Hopko D.R., R. Mahadevan, R.L. Bare, et al. 2003. The abbreviated math anxiety scale (AMAS) construction, validity, and reliability. Assessment 10: 178-182. 
54. Morsanyi K., I.C. Mammarella, D. Szücs, et al. 2016. Editorial: Mathematical and statistics anxiety: Educational, social, developmental and cognitive perspectives. Frontiers in Psychology 7: 1-4.

55. Ashcraft M.H. \& M.W. Faust. 1994. Mathematics anxiety and mental arithmetic performance: An exploratory investigation. Cognition \& Emotion 8: 97-125.

56. Ashcraft M.H. \& A.M. Moore. 2009. Mathematics anxiety and the affective drop in performance. Journal of Psychoeducational Assessment 27: 197-205.

57. Daker R.J., S.U. Gattas, H.M. Sokolowski, et al. 2021. First-year students' math anxiety predicts STEM avoidance and underperformance throughout university, independently of math ability. npj Science of Learning 2021 6:1 6: 1-13.

58. Pollack C., D. Wilmot, T.M. Centanni, et al. 2021. Anxiety, Motivation, and Competence in Mathematics and Reading for Children With and Without Learning Difficulties. Frontiers in Psychology 12: 4193.

59. Wang Z., S.L. Lukowski, S.A. Hart, et al. 2015. Is Math Anxiety Always Bad for Math Learning? The Role of Math Motivation. Psychological Science 26: 1863-1876.

60. Orbach L. \& A. Fritz. 2021. A latent profile analysis of math anxiety and core beliefs toward mathematics among children. Annals of the New York Academy of Sciences.

61. Meier M.A., S.E. Vogel \& R.H. Grabner. 2021. Going Beyond Intelligence: A Systematic Investigation of Cognitive Abilities and Personality Traits of Experts in Mathematics. Journal of Expertise 4: 80-115.

62. Ramirez G. \& S.L. Beilock. 2011. Writing about testing worries boosts exam performance in the classroom. Science 331: 211-213.

63. Jamieson J.P., W.B. Mendes, E. Blackstock, et al. 2010. Turning the knots in your stomach into bows: Reappraising arousal improves performance on the GRE. Journal of Experimental Social Psychology 46: 208-212.

64. Ganley C.M., R.A. Conlon, A.L. McGraw, et al. 2021. The effect of brief anxiety interventions on reported anxiety and math test performance. Journal of Numerical Cognition 7: 4-19.

65. Moustafa A.A., A.A. Al-Emadi \& A.M. Megreya. 2021. The Need to Develop an Individualized Intervention for Mathematics Anxiety. Frontiers in Psychology 12: 4396.

66. Dondio P., F.H. Santos, V. Gusev, et al. 2021. Do Games Reduce Maths Anxiety? A Review of the Current Literature. In ECGBL 2021-Proceedings of the 15th European Conference on Game Based Learning Fotaris P., Ed. Brighton, UK: ACI.

67. Artemenko C., N. Masson, C. Georges, et al. 2021. Not all elementary school teachers are scared of math. Journal of Numerical Cognition.

68. Costa H.M., L.A. Outhwaite \& J. van Herwegen. 2021. Preschool Teachers' training, beliefs and practices concerning mathematics in pre-schools in the UK: implication for education and practice. Psyarxiv.

69. Stoehr K.J. \& A.M. Olson. 2021. Elementary prospective teachers' visions of moving beyond mathematics anxiety. Mathematics Education Research Journal 1-20.

70. Olson A.M. \& K.J. Stoehr. 2019. From numbers to narratives: Preservice teachers experiences' with mathematics anxiety and mathematics teaching anxiety. School Science and Mathematics 119: 72-82.

71. Beilock S.L., E.A. Gunderson, G. Ramirez, et al. 2010. Female teachers' math anxiety affects girls' math achievement. Proceedings of the National Academy of Sciences 107: 1860-1863. 
72. DiStefano M., B. O'Brien, A. Storozuk, et al. 2020. Exploring math anxious parents' emotional experience surrounding math homework-help. International Journal of Educational Research 99: 101526.

73. Maloney E.A., G. Ramirez, E.A. Gunderson, et al. 2015. Intergenerational effects of parents' math anxiety on children's math achievement and anxiety. Psychological Science 26: 1480-1488.

74. Vanbinst K., E. Bellon \& A. Dowker. 2020. Mathematics Anxiety: An Intergenerational Approach. Frontiers in Psychology 11: 1648.

75. Hunt T.E., D. Clark-Carter \& D. Sheffield. 2011. The development and part validation of a U.K. scale for mathematics anxiety. Journal of Psychoeducational Assessment 29: 455-466.

76. Evans J. 2000. "Adults' Mathematical Thinking and Emotions: A Study of Numerate Practices." London: Routledge.

77. Pletzer B., G. Wood, T. Scherndl, et al. 2016. Components of mathematics anxiety: Factor modeling of the MARS30-brief. Frontiers in Psychology 7: 1-14.

78. OECD. 2010. "The high cost of low educational performance: The long-run economic impact of improving educational outcomes." Paris: OECD.

79. Butterworth B., S. Varma \& D. Laurillard. 2011. Dyscalculia: From brain to education. Science 332: 1049-1053.

80. Avey J.B., T.S. Wernsing \& K.H. Mhatre. 2011. A Longitudinal Analysis of Positive Psychological Constructs and Emotions on Stress, Anxiety, and Well-Being: http://dx.doi.org/10.1177/1548051810397368 18: 216-228.

81. Haase V.G., A.P.L. Guimarães \& G. Wood. 2019. Mathematics and Emotions: The Case of Math Anxiety. International Handbook of Mathematical Learning Difficulties: From the Laboratory to the Classroom 469-503.

82. Wu S.S., E.G. Willcutt, E. Escovar, et al. 2014. Mathematics Achievement and Anxiety and Their Relation to Internalizing and Externalizing Behaviors. Journal of Learning Disabilities 47: 503-514.

83. Paidar F., A. Amirhooshangi \& R. Taghavi. 2017. Gender Differences in Students' Mathematics Self-Concept and Academic Burnout. International Journal of School Health 4: 1-6.

84. Marin M.F., C. Lord, J. Andrews, et al. 2011. Chronic stress, cognitive functioning and mental health. Neurobiology of Learning and Memory 96: 583-595.

85. Ellis J., B.K. Fosdick \& C. Rasmussen. 2016. Women 1.5 Times More Likely to Leave STEM Pipeline after Calculus Compared to Men: Lack of Mathematical Confidence a Potential Culprit. PLOS ONE 11: e0157447. 